\title{
APPLICATION OF THE NONUNIFORM FAST FOURIER TRANSFORM TO THE DIRECT NUMERICAL SIMULATION OF TWO-WAY COUPLED PARTICLE LADEN FLOWS
}

\author{
MAURIZIO CARBONE \& MICHELE IOVIENO \\ Politecnico di Torino, Dipartimento di Ingegneria Meccanica e Aerospaziale, 10129 Torino, Italy
}

\begin{abstract}
We present the application of the Nonuniform Fast Fourier Transform (NUFFT) to the pseudo-spectral Eulerian-Lagrangian direct numerical simulation of particle-laden flows. In the two-way coupling regime, when the particle feedback on the flow is taken into account, a spectral method requires not only the interpolation of the flow fields at particle positions, but also the Fourier representation of the particle back-reaction on the flow fields on a regular grid. Even though the direct B-spline interpolation is a well-established tool, to the best of our knowledge the reverse projection scheme has never been used, replaced by less accurate linear reverse interpolation or Gaussian regularization. We propose to compute the particle momentum and temperature feedback on the flow by means of the forward NUFFT, while the backward NUFFT is used to perform the B-spline interpolation. Since the backward and forward transformations are symmetric and the (non local) convolution computed in physical space is removed in Fourier space, this procedure satisfies all constraints for a consistent interpolation scheme, and allows an efficient implementation of high-order interpolations. The resulting method is applied to the direct numerical simulation of a forced and isotropic turbulent flow with different particle Stokes numbers in the two-way coupling regime. A marked multifractal scaling is observed in the particle statistics, which implies that the feedback from the particles on the fields is far from being analytic and therefore only high-order methods, like the one here proposed, can provide an accurate representation.
\end{abstract}

Keywords: turbulence, particle laden flows, two-way coupling, direct numerical simulation.

\section{INTRODUCTION}

Inertial particles in turbulence play a critical role in many fields, ranging from climate physics, astrophysics and engineering. Applications include cyclonic separators of industrial plants, sprays for surface coating, combustion chambers of internal combustion engines and cooling of miniaturized components [1]. Many works in the literature focused on the one-way coupling regime [2], that is, the flow drives the particles dynamics but the back-reaction of the particles on the fluid is neglected. In that limit, a variety of intriguing phenomena arise such as clustering and caustics [3], [4]. However, in many physical systems and applications the particle volume fraction is high enough that the particle dynamics significantly modifies the features of the flow.

Direct Numerical Simulation (DNS) is a key instrument to gain insight into particleladen turbulent flows. The most common approach is the solution of the fluid flow on a Eulerian structured grid with Lagrangian tracking of the dispersed particles [5]. This approach requires the computation of the fields at the particle position and the representation of the coupling terms on the Eulerian grid. Well established and efficient algorithms are available for the interpolation of the fields at the particle position [6], [7]. On the other hand, a few techniques are available to accurately represent the back-reaction of the particles on the flow at the grid points. Only recently a systematic study of the convergence and accuracy of the "reverse interpolation" methods was carried out for a few algorithms [8]. In this paper we propose to use the Non Uniform Fast Fourier Transform (NUFFT) as a tool to compute the coupling term in the direct numerical 
simulation of dilute suspensions of point-like inertial particles in turbulence. The NUFFT turns out to be an accurate and efficient method, especially suitable for pseudo-spectral codes.

The NUFFT has been used in a wide range of applications, from medical imaging [9] to molecular dynamics [10], [11]. By means of a discrete convolution and fast Fourier transform over an oversampled grid, NUFFT provides a representation of the Fourier transform of an irregular and non-uniformly sampled field. The number of operations required for the computation of the NUFFT with accuracy $\varepsilon$ of a field sampled at $M$ points is $\mathcal{O}(M \log (M)-M \log (\varepsilon))$ [12]. The NUFFT consists of three steps: (1) a convolution in physical space of the field with a basis function to regularize the field; (2) FFT of the regularized field; (3) a deconvolution in Fourier space. Accuracy is determined by the properties of the basis function used for the convolution and by the smoothness of the field. In the point-mass approximation the particle back-reaction is described by a superposition of Dirac delta functions centered on the particles. According to the classification by [13], a type-1 NUFFT is used to compute the coupling and a type-2 NUFFT is used to interpolate. The B-spline interpolation (type-2 NUFFT) performs better than the other methods currently available [7], especially if employed along with pseudo-spectral methods. The type-1 NUFFT is nothing but the inverse type-2 NUFFT, therefore we expect that the type-1 NUFFT enjoys the same benefits as the B-spline interpolation.

The interpolation and reverse interpolation methods should satisfy a few constraints. By accurate consideration on the energy balance of the coupled system Sundaram and Collins [14] pointed out that the interpolation schemes for interpolation and reverse interpolation must be symmetric in order to guarantee the energy conservation. Furthermore, they argued that the spurious non-locality introduced by interpolation and reverse interpolation should be minimized for a consistent and convergent method. These constraints are naturally satisfied by the B-spline interpolation (regarded as a backward NUFFT) together with the forward NUFFT for the computation of the coupling. Indeed, the backward NUFFT is the inverse of the forward NUFFT and they are symmetric by definition. Moreover, the non-locality introduced in physical space is removed in Fourier space because convolution and deconvolution are carried out with respect to the same basis. Finally, the localized support of the B-spline basis allows the efficient parallelization of the NUFFT algorithm, since the particle affects only a limited region of the surrounding flow and next neighbor communications suffice [11], an important feature of any algorithm devoted to the direct numerical simulation of turbulent flows.

In principle, the NUFFT presents several advantages with respect to the other methods reported in the literature. The particle in cell (PIC) method is a straightforward approach to deal with the coupling term. The force exerted by the particle on the surrounding fluid is distributed over the surrounding grid points of the cell in which the particle is located, as in the reverse linear interpolation [15]. Therefore the accuracy of PIC methods intrinsically depends on the particle number density. Regularization functions usually exploited to represent the Dirac delta [16] guarantee numerical stability but damp high frequencies and the resulting coupling terms depend on the regularization scale. Steady Stokeslet method [17] can overcome the grid dependence but the disturbance decays slowly with the distance from the particle, therefore a large number of operations is necessary and parallel implementation is challenging. Gualtieri et al. [8] exploited the closed solution of the unsteady Stokes flow around a small rigid sphere in order to isolate the singular and regular part of the flow at scales smaller than the grid resolution. This Exact Regularized Point Particle method (ERPP) guarantees high accuracy and the momentum conservation, however, it requires the evaluation of the analytical solution for the unsteady Stokes flow at a previous time, at each time step. 
Most of the works in the literature separated the analysis of the algorithm from the physics of inertial particles in turbulence. However, an intriguing scenario arises when the performance of the NUFFT algorithm is analyzed as a function of particle inertia because the accuracy of the method depends on the regularity of the coupling term which in turns depends on the particle inertia. In particular, we show that the statistics of the coupling term, such as its energy spectrum, are intrinsically related to the two-particle statistics.

\subsection{The physical model}

\section{METHODOLOGY}

We employ the Eulerian-Lagrangian point particle approach in the two-way coupling regime, where the transported particles are material point masses which exchange momentum and heat with the fluid phase. By considering the fluid temperature field as an advected passive scalar, the equations for the fluid phase are

$$
\begin{aligned}
\nabla \cdot \mathbf{u} & =0 \\
\partial_{t} \mathbf{u}+\nabla \cdot\left(\mathbf{u u}^{\top}\right) & =-\frac{1}{\rho_{(f)}} \nabla p+\nu \nabla^{2} \mathbf{u}-\mathbf{C}_{\mathbf{u}}+\mathbf{f}, \\
\partial_{t} T+\nabla \cdot(\mathbf{u} T) & =\kappa \nabla^{2} T-C_{T}+f_{T} .
\end{aligned}
$$

Here $\rho_{(f)}, T(\mathbf{x}, t)$ and $\mathbf{u}(\mathbf{x}, t)$ are the fluid density, temperature and velocity, $p(\mathbf{x}, t)$ is the pressure, $\nu$ is its kinematic viscosity and $\kappa$ is the thermal conductivity; the $\mathbf{C}_{\mathbf{u}}$ and $C_{T}$ terms are the momentum and heat feedback of the particles on the flow and $\mathbf{f}, f_{T}$ represent a large scale external forcing, which keeps constant the dissipation rate of turbulent kinetic energy $\varepsilon$ and of temperature variance $\chi$. In wavenumber space the forcing is defined as

$$
\hat{\mathbf{f}}(\mathbf{k}, t)=\varepsilon \frac{\hat{\mathbf{u}}(\mathbf{k}, t)}{\sum_{\mathbf{k} \in \mathcal{K}_{f}}\|\hat{\mathbf{u}}(\mathbf{k}, t)\|^{2}}, \quad \hat{f}_{T}(\mathbf{k}, t)=\chi \frac{\hat{T}(\mathbf{k}, t)}{\sum_{\mathbf{k} \in \mathcal{K}_{f}}|\hat{T}(\mathbf{k}, t)|^{2}},
$$

where the hat indicates the Fourier transform and $\mathcal{K}_{f}$ is the set of forced wavenumbers, as to mimic a large scale Gaussian forcing [15]. The flow is seeded by small, sub-Kolmogorov, spherical heavy particles with radius $r_{(p)}$ and density $\rho_{(p)}$, whose dynamics, is described by a simplified version of the Maxey and Riley equation [18] and a linear Newton law for heat conduction, that is, by the following system

$$
\begin{aligned}
\frac{\mathrm{d} \mathbf{x}_{(p)}}{\mathrm{d} t} & =\mathbf{v}_{(p)}, \\
\frac{\mathrm{d} \mathbf{v}_{(p)}}{\mathrm{d} t} & =\frac{\mathbf{u}\left(\mathbf{x}_{(p)}, t\right)-\mathbf{v}_{(p)}}{\tau_{u,(p)}}, \\
\frac{\mathrm{d} \theta_{(p)}}{\mathrm{d} t} & =\frac{T\left(\mathbf{x}_{(p)}, t\right)-\theta_{(p)}}{\tau_{\theta,(p)}} .
\end{aligned}
$$

Here $\tau_{u,(p)}$ and $\tau_{\theta,(p)}$ are the particle momentum and thermal response time, which, for $\rho_{(p)} \gg \rho_{(f)}$, are given by

$$
\tau_{u,(p)}=\frac{2}{9} \frac{\rho_{(p)}}{\rho_{(f)}} \frac{r_{(p)}^{2}}{\nu}, \quad \tau_{\theta,(p)}=\frac{1}{3} \frac{\rho_{(p)}}{\rho_{(f)}} \frac{c_{(p)}}{c_{f}} \frac{r_{(p)}^{2}}{\kappa},
$$


where $c_{(p)}$ and $c_{(f)}$ are the particle and fluid specific heat capacity at constant pressure. The Stokes number and the thermal Stokes number are defined as $S t=\tau_{u,(p)} / \tau_{\eta}, S t_{\theta}=$ $\tau_{\theta,(p)} / \tau_{\eta}$, where $\tau_{\eta}$ is the Kolmogorov time scale.

When the fluid exerts a force of the particle the particles exerts an opposite force on the fluid at the particle position. Analogously, when heat flows from the fluid to the particle the fluid loses thermal energy at the particle position. This feedback from the particles on the fields is modeled by means of a superposition of Dirac delta functions centered on the particles

$$
\begin{aligned}
& \mathbf{C}_{\mathbf{u}}(\mathbf{x}, t)=\frac{4}{3} \pi \frac{\rho_{(p)}}{\rho_{(f)}} \sum_{p=1}^{N_{(P)}} r_{(p)}^{3} \frac{\mathrm{d} \mathbf{v}_{(p)}}{\mathrm{d} t} \delta\left(\mathbf{x}-\mathbf{x}_{(p)}\right) \\
& C_{T}(\mathbf{x}, t)=\frac{4}{3} \pi \frac{\rho_{(p)}}{\rho_{(f)}} \frac{c_{(p)}}{c_{(f)}} r_{(p)}^{3} \sum_{p=1}^{N_{(P)}} \frac{\mathrm{d} \theta_{(p)}}{\mathrm{d} t} \delta\left(\mathbf{x}-\mathbf{x}_{(p)}\right)
\end{aligned}
$$

The force exerted by the particle on the finite region of fluid $\Omega^{\prime}$, centered at $\mathbf{x}$ is

$$
\widetilde{\mathbf{C}_{\mathbf{u}}}(\mathbf{x}, t)=\frac{4}{3} \pi \frac{\rho_{(p)}}{\rho_{(f)}} \frac{r_{(p)}^{3}}{\left|\Omega^{\prime}\right|} \sum_{\mathbf{x}_{p} \in \Omega^{\prime}(\mathbf{x})} \frac{\mathrm{d} \mathbf{v}_{(p)}}{\mathrm{d} t}
$$

The coarse grained back-reaction, eqn (4), is exploited to analyze the regularity of the coupling terms, by characterizing its increments $\delta_{\mathbf{r}} \widetilde{\mathbf{C}_{\mathbf{u}}}(\mathbf{x}, t)=\widetilde{\mathbf{C}_{\mathbf{u}}}(\mathbf{x}+\mathbf{r}, t)-\widetilde{\mathbf{C}_{\mathbf{u}}}(\mathbf{x})$ using the Hölder condition

$$
\left\langle\left\|\delta_{\mathbf{r}} \mathbf{C}_{\mathbf{u}}\right\|\right\rangle \leq \Lambda\|\mathbf{r}\|^{\alpha_{H}}, \forall \mathbf{x}, \mathbf{r} \in \Omega
$$

for non-negative real numbers $\Lambda$ and $\alpha_{H}$, independent of $\mathbf{x}$ and $\mathbf{r}$. Here $\langle\cdot\rangle$ is the ensemble average, approximated by a spatial average for isotropic turbulence. Eqn (4) shows that the increments of the coarse-grained coupling field are proportional to the increments of the particle acceleration $\left\langle\delta_{\mathbf{r}}\left\|\mathbf{C}_{\mathbf{u}}\right\|\right\rangle \propto\left\langle\delta_{\mathbf{r}}\|\dot{\mathbf{v}}\|\right\rangle$, thus the structure functions of the particle acceleration provide insight on the regularity of the coupling field. In particular, they highlight the dependence on the particle inertia of the order of Hölder-continuity $\alpha_{H}$. At small separation, the particle acceleration structure functions show a well defined power law behaviour $\left\langle\delta_{r}\|\dot{\mathbf{v}}\|\right\rangle \sim r^{\alpha(S t)}$, as in Fig. 4, which implies

$$
\left\langle\delta_{r}\left\|\mathbf{C}_{\mathbf{u}}\right\|\right\rangle \sim r^{\alpha(S t)} \leq \Lambda r^{\alpha_{H}}
$$

for $r \rightarrow 0$. Hence, the scaling exponent of the structure functions at small separation $\alpha(S t)$ is an upper bound for $\alpha_{H}$. When the particle collisions are not taken to account and the ghost collision approximation is used, particles may overlap breaking the relation $\left\langle\delta_{\mathbf{r}}\left\|\mathbf{C}_{\mathbf{u}}\right\|\right\rangle \propto\left\langle\delta_{\mathbf{r}}\|\dot{\mathbf{v}}\|\right\rangle$. However, we expect that in a steady and isotropic turbulent flow the exact overlapping of distinct particles is an event of zero probability.

\subsection{The numerical method}

The Navier-Stokes eqns (1) are solved in a tri-periodic cubic domain $\Omega=[0,2 \pi]^{3}$ by means of a pseudo-spectral Fourier method. The $3 / 2$ rule is employed for the dealiasing of the convolution sums [19] therefore, the number of grid points in each direction is $N$ in Fourier space and $M=3 N / 2$ in physical space. The fluid velocity and temperature are integrated 
in time by means of a second order exponential Runge-Kutta method [20], which has been preferred to the standard integrating factor for its unconditioned consistency, since the conservation of the total kinetic and thermal energy is a critical aspect of the simulation. The same method is used to integrate particle equations. This guarantees consistency, since the system formed by particles and fluid is evolved in time as a whole.

\subsubsection{The B-spline polynomial basis}

Let us consider the B-spline polynomial $B_{N_{S}-1}$ of order $N_{S}-1$. The one dimensional Bspline polynomials are defined by the convolution $B_{N_{S}}(x)=B_{N_{S}-1}(x) * B_{0}(x)$ where $B_{0}$ is the rectangular function defined on an interval of amplitude $\Delta x$ (that is the grid spacing in the numerical implementation)

$$
B_{0}(x) \Delta x= \begin{cases}1, & \text { if }|x| / \Delta x \leq 1 / 2 \\ 0, & \text { otherwise }\end{cases}
$$

so that the Fourier representation of the B-spline basis is

$$
\widehat{B}_{N_{S}-1}\left(k_{x}\right)=\frac{1}{2 \pi}\left(\operatorname{sinc}\left(k_{x} \frac{\Delta x}{2}\right)\right)^{N_{S}} \text {. }
$$

The spline basis in three dimensions is obtained by separation of variables [7], $B(\mathbf{x})=$ $B(x) B(y) B(z)$. For sake of clarity, the order subscript will be omitted. This basis guarantees consistency, since $|\Omega| \widehat{B}(\mathbf{0})=1$, and its Fourier transform can be pre-computed and stored. A most important computational feature is the localized support of the B-spline basis, since the convolutions in physical space are executed on a limited portion of the domain, thus allowing an efficient parallelization [11]. More detailed description of the properties of the B-spline polynomials in the framework of NUFFT can be found in [21].

\subsubsection{Interpolation: backward NUFFT}

The interpolation is carried out as a backward Non Uniform Fourier Transform with Bspline basis, and consists of three steps [7], [13]. First, a field $u(\mathbf{x})-$ a velocity component or a transported scalar - is projected onto the B-spline basis in Fourier space, by means of a deconvolution which is efficiently carried out in the wavenumber space, $\widehat{\widetilde{u}}(\mathbf{k})=$ $\widehat{u}(\mathbf{k}) /(|\Omega| \widehat{B}(\mathbf{k}))$. In a spectral DNS, due to the dealiasing, $\left|k_{x} \Delta x\right| \leq 2 \pi / 3$ so that $\widehat{B}$, defined in eqn (5), is always positive. Then an inverse FFT transforms $\widehat{\widetilde{u}}(\mathbf{k})$ to the physical space. Finally the field $\widetilde{u}(\mathbf{x})$ is interpolated at each particle position by means of a convolution in physical space:

$$
u\left(\mathbf{x}_{(p)}\right)=\int_{\Omega_{B}} B\left(\mathbf{x}-\mathbf{x}_{(p)}\right) \widetilde{u}(\mathbf{x}) \mathrm{d} \mathbf{x},
$$

where $\Omega_{B}$ is the support of the B-spline polynomial. This convolution in physical space and the former deconvolution in Fourier space cancel out, resulting in an accurate representation of the actual field at the particle position. Without the deconvolution in Fourier space, the interpolated field would be coarse-grained and its value at the particle position would be affected by points which are far away from the particle [14]. 


\subsubsection{Reverse interpolation: forward NUFFT}

The Fourier transform of the momentum or heat back-reaction of the particles on the fluid, represented by a sum of Dirac delta functions as in eqn (3), is carried out by means of a forward NUFFT with B-spline basis. The forward NUFFT consists of three steps [13]. At first, the comb $C(\mathbf{x})$ - a component of the momentum coupling or the temperature coupling - is distributed on the surrounding grid points according to the B-spline weights. The convolution of the superposition of spikes with the B-spline polynomials results into the regularized field

$$
\widetilde{C}(\mathbf{x})=\frac{4}{3} \pi \frac{\rho_{(p)}}{\rho_{(f)}} r_{(p)}^{3} \sum_{p} \dot{v}_{(p)} \int_{\Omega_{B}} \delta\left(\mathbf{y}-\mathbf{x}_{(p)}\right) B(\mathbf{x}-\mathbf{y}) \mathrm{d} \mathbf{y} .
$$

Then the FFT is applied to the regularized field $\widetilde{C}$ and, finally, the deconvolution is carried out in the wavenumber space,

$$
\widehat{C}(\mathbf{k})=\frac{1}{|\Omega|} \frac{\widehat{\widetilde{C}}(\mathbf{k})}{\widehat{B}(\mathbf{k})}
$$

to remove the coarse-graining, thus avoiding the non-locality of the interpolation. This step can be interpreted as gathering together the sub-particles obtained splitting the actual particle in the first step, eqn (7). Hence, the NUFFT does not introduce non-locality and the symmetry of forward and backward NUFFTs guarantees energy conservation [14].

\subsubsection{Implementation}

The discrete convolution required to carry out the NUFFTs is discretized as

$$
u\left(\mathbf{x}_{(p)}\right)=\int_{\Omega_{B}} B\left(\mathbf{x}-\mathbf{x}_{(p)}\right) \widetilde{u}(\mathbf{x}) \mathbf{d} \mathbf{x} \approx \sum_{l, m, n} \Delta x^{3} B\left(\mathbf{x}_{l m n}-\mathbf{x}_{(p)}\right) \widetilde{u}_{l m n}
$$

where the components of the position vector $\mathbf{x}_{l m n}$ in the Cartesian basis are $\Delta x[l, m, n]^{\top}$, $\widetilde{u}_{l m n}=\widetilde{u}\left(\mathbf{x}_{l m n}\right)$ and the sum is extended over the support of the B-spline. Separation of variables is exploited to reduce the number of operations, executing the contraction of the three indexes as a sequence of three contractions on each index [7]. Analogously, the continuous convolution in physical space with the B-spline basis applied to the coupling term, eqn (7), results into the discrete sum

$$
\widetilde{C}_{l m n}=\frac{4}{3} \pi \frac{\rho_{(p)}}{\rho_{(f)}} \frac{r_{(p)}^{3}}{\Delta x^{3}} \sum_{p} \dot{v}_{(p)} \Delta x^{3} B\left(\mathbf{x}_{l m n}-\mathbf{x}_{(p)}\right) .
$$

The discrete convolution in physical space virtually splits each particle in many sub-particles located at the neighbouring grid points. The mass and thermal energy of the sub-particles located at $\mathbf{x}_{l m n}$ is $\Delta x^{3} B\left(\mathbf{x}_{l m n}-\mathbf{x}_{(p)}\right)$ times smaller than the mass of the actual particle. The fictitious particles undergo the same acceleration/temperature variation of the actual particle and are gathered in Fourier space, as in eqn (8), to give back the actual particle. The contribution $\widetilde{C}_{l m n(p)}$ to the coupling term $\widetilde{C}_{l m n}$ from the particle $(p)$ is computed using the algorithm reported in [7] in reverse, exploiting again separation of variables,

$$
\begin{aligned}
\widetilde{C}_{l(p)} & =\frac{4}{3} \pi \frac{\rho_{(p)}}{\rho_{(f)}} r_{(p)}^{3} B_{N_{S}}\left(x_{l}-x_{(p)}\right) \dot{v}_{(p)}, \\
\widetilde{C}_{l m(p)} & =B_{N_{S}}\left(y_{m}-y_{(p)}\right) \widetilde{C}_{l(p)},
\end{aligned}
$$




$$
\widetilde{C}_{l m n(p)}=B_{N_{S}}\left(z_{n}-z_{(p)}\right) \widetilde{C}_{l m(p)}
$$

with a noticeable speed-up of the computation. The Eulerian-Lagrangian code is parallelized by means of the Message Passing Interface with a two dimensional (pencil) domain decomposition.The P3DFFT library [22] is employed to carry out the three-dimensional FFT in parallel. The same Eulerian pencil decomposition is employed for the particles: particles travel through the domain as the simulation advances and they are exchanged between neighbouring processors when they cross the processor boundary. Communication is required to share only a tiny part of the field data at the boundary between two processors.

\section{RESULTS}

\subsection{Convergence and accuracy}

As a preliminary test, we have examined the convergence of our implementation of the NUFFT for different order of the B-spline basis, that is, varying the number of points used for the discrete convolution, eqns (9)-(10). To assess the accuracy of the B-spline interpolation [7], computed as a backward NUFFT, the following set of three-dimensional trigonometric polynomials with unitary root mean square value in $\Omega$ has been used,

$$
T_{k}=\left\{\overline{f_{k}^{j}}(\mathbf{x})=\sqrt{8} \cos \left(k_{x} x\right) \sin \left(k_{y} y\right) \cos \left(k_{z} z\right):\left\lfloor k_{x}^{2}+k_{y}^{2}+k_{z}^{2}\right\rceil=k^{2}\right\} .
$$

$T_{k}$ is a subset of the trigonometric polynomials with wavevector in the shell $\|\mathbf{k}\|=k$ so it contains $N_{T_{k}} \approx 4 \pi k^{2}$ elements. The backward NUFFT takes as input the polynomial $\overline{f_{k}^{j}}$ together with the particle position $\mathbf{x}_{(p)}$ and returns the approximation $f_{k}^{j}\left(\mathbf{x}_{(p)}\right)$ of the value of the polynomial at the particle position. The error of the backward NUFFT can be estimated as the average of the absolute errors occurred in the interpolation of each element $f_{k}^{j}$ of $T_{k}$, averaged over all the particles:

$$
\epsilon_{k}^{b}=\frac{1}{N_{(P)}} \frac{1}{N_{T_{k}}} \sum_{p=1}^{N_{(P)}} \sum_{j=1}^{N_{T_{k}}}\left|f_{k}^{j}\left(\mathbf{x}_{(p)}\right)-\overline{f_{k}^{j}}\left(\mathbf{x}_{(p)}\right)\right| .
$$

The convergence and accuracy of the forward NUFFT with B-spline basis is assessed by performing the Fourier transform of a three-dimensional random field. The test signal is a superposition of Dirac delta functions with random, uniformly distributed, amplitude $a_{(p)}$ centered at random points $\mathbf{x}_{(p)}$,

$$
C(\mathbf{x})=\sum_{p} a_{(p)} \delta\left(\mathbf{x}-\mathbf{x}_{(p)}\right)
$$

The mean amplitude of the spikes is zero, while its variance is unitary. The forward NUFFT takes as input the positions of the particles together with the amplitude of the spikes and returns the approximation $\widehat{C}_{\mathbf{k}}$ of the Fourier transform of the superposition of Dirac functions. We define the error of the forward NUFFT as the average of the absolute errors occurred in the computation of each mode of the Fourier transform $\widehat{C}_{\mathbf{k}}$, averaged over all the modes at constant $k$ :

$$
\epsilon_{k}^{f}=\frac{1}{N_{T_{k}}} \sum_{j=1}^{N_{T_{k}}} \mid \widehat{C}_{\mathbf{k}}^{j}-{\overline{\widehat{C}_{\mathbf{k}}^{j}}}_{\|\mathbf{k}\|=k} .
$$



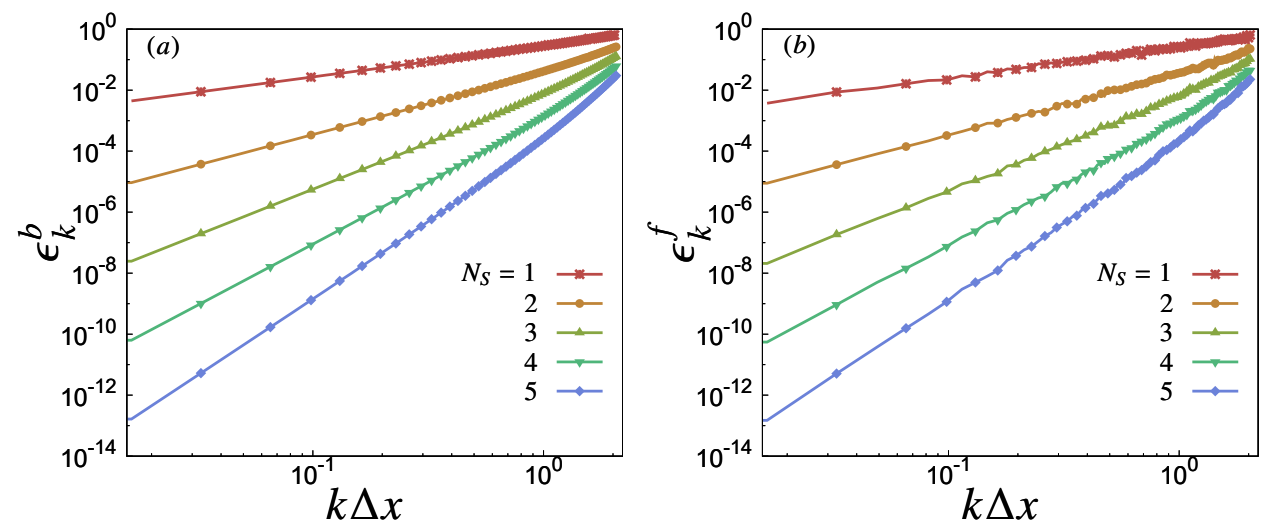

Figure 1: Absolute error of the NUFFT for different degrees of the polynomial basis. (a) Error in the interpolation of a trigonometric polynomial with unitary rms value and wavenumber $k$; (b) Error in the Fourier transform of a random uniform field with unitary rms value at wavenumber $k$.
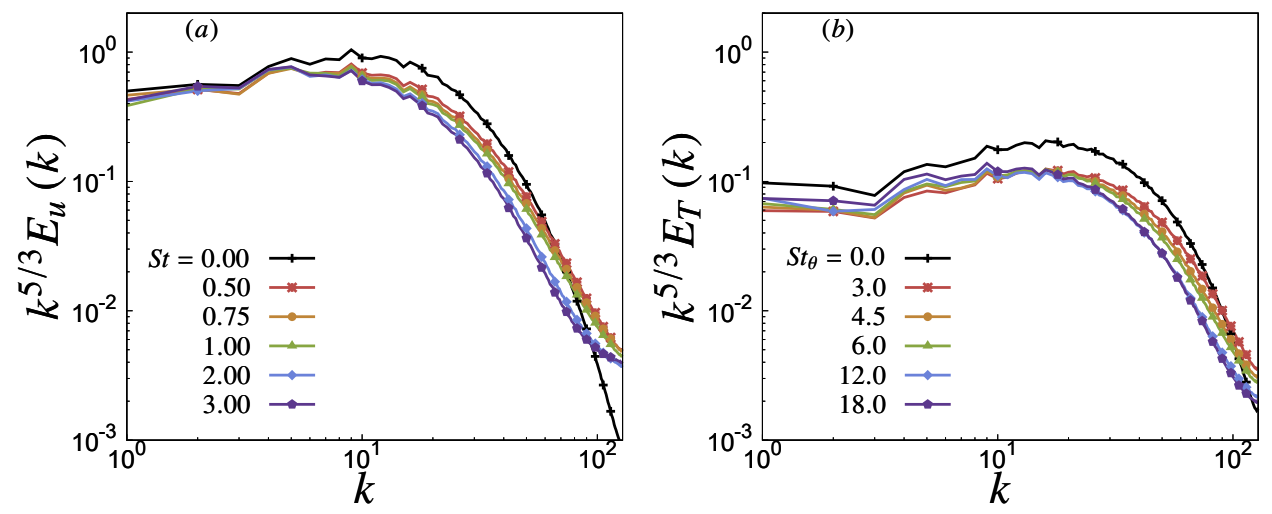

Figure 2: Spectra of the flow fields for different particle inertia, measured by the Stokes number. (a) Fluid velocity spectra; (b) Fluid temperature spectra.

Fig. 1 shows the error of the backward NUFFT $\epsilon_{k}^{b}$ and the forward NUFFT $\epsilon_{k}^{f}$ for different degrees of the B-spline polynomial basis. The order of convergence matches the number of points used for the interpolation in each direction: $\epsilon_{k}^{b} \sim k^{-N_{S}}$ for $k \rightarrow 0$. It is critical to introduce a small error at low wavenumbers, since the large scales of the flow contain the largest amount of the energy in a turbulent flow, as shown in Fig. 2. The optimum value of the order of interpolation for the DNS of particle-laden turbulent flows has been discussed in [23]. Remarkably, the absolute errors of the backward and forward NUFFT are the same. This fact assesses the forward NUFFT as an accurate and efficient tool for computing the back-reaction of the particles on the flow. Indeed, the forward NUFFT shares all the advantages of the Bspline interpolation (backward NUFFT) which performs vastly better than other methods [7], especially if employed in pseudo-spectral codes. 


\subsection{Direct numerical simulation of isotropic steady turbulence}

We present some preliminary results which characterize the effect of the particle inertia on the turbulence statistics. The parameters used in the simulations are in Table 1. A first tool to gain insight in to the dynamics of the system is the energy balance [14], which for isotropic steady turbulence reduces to

$$
\begin{aligned}
& \varepsilon=\nu\left[\left\langle\|\nabla \mathbf{u}\|_{F}^{2}\right\rangle+\frac{9}{2} \phi\left\langle\frac{\left\|\mathbf{v}_{(p)}-\mathbf{u}_{(p)}\right\|^{2}}{r_{(p)}^{2}}\right\rangle\right], \\
& \chi=\kappa\left[\left\langle\|\nabla T\|^{2}\right\rangle+3 \phi\left\langle\frac{\left(\theta_{(p)}-T_{(p)}\right)^{2}}{r_{(p)}^{2}}\right\rangle\right],
\end{aligned}
$$

where $\phi=4 / 3 \pi r_{(p)}^{3} N_{(P)} /|\Omega|$ is the particle volume fraction. In steady turbulence, the rate of energy injection by the external forcing balances the overall dissipation rate, which is the sum of the fluid and particle dissipation since, as eqn (11) shows, particles always dissipate energy in the limit of the point particle approximation. Therefore, the accuracy of the computation of the particle back-reaction on the flow directly affects the energy balance. The energy spectrum of the velocity and temperature fields for different particle inertia are shown in Fig. 2. The particles, through their back-reaction, significantly modify the spectral distribution of the energy of the fields. Particles with high inertia strongly smooth out the gradients of the fields. However, they carry energy from the large scales to the high wavenumbers by means of their inertia.

In order to assess the convergence of the method we consider a snapshot of the field and the particles and we compute the spectra of the coupling terms using B-spline polynomial basis of different degrees. In the previous section we have shown that the NUFFT of a random field rapidly converges towards the exact direct Fourier transform as the degree of the basis polynomial is increased. The global error can be estimated from the spectrum obtained with

Table 1: Parameters, in dimensionless code units, of the forced turbulence test case.

\begin{tabular}{lcc}
\hline Dimensionless kinematic viscosity & $\nu$ & 0.002 \\
Schmidt number & $S c$ & 1 \\
TKE dissipation rate & $\varepsilon$ & 0.27 \\
Temperature fluctuation & $\chi$ & 0.1 \\
dissipation rate & $R e_{\lambda}$ & 140 \\
Reynolds number & $k_{f}$ & $\sqrt{2}$ \\
Forced wavenumber & $N$ & 256 \\
Grid size & $k_{\max } \eta$ & 1.68 \\
Resolution & $\rho_{(p)} / \rho_{(f)}$ & 1000 \\
\hline Particle/fluid density ratio & $c_{(p)} / c_{(f)}$ & 4 \\
Particle/fluid specific heat capacity ratio & $\phi$ & 0.0002 \\
Volume fraction & $S t$ & $0.5,0.75,1,1.5,2,3$ \\
Stokes number & $S t_{\theta}$ & $6 S t$ \\
Thermal Stokes number & & \\
\hline
\end{tabular}


different order basis,

$$
\epsilon^{s}\left(N_{S}\right)=\frac{\left.\sum_{k}|| \widehat{C}_{k}\right|_{N_{S}} ^{2}-\left|\widehat{C}_{k}\right|_{N_{S}-1}^{2} \mid}{\sum_{k}\left|\widehat{C}_{k}\right|_{N_{S}}^{2}},
$$

where subscript $N_{S}$ indicates the number of points used for the discrete convolution. Fig. 3 shows the spectrum of the momentum coupling for different particle inertia and the convergence of the spectrum of the coupling increasing the order of the basis. The relative error $\epsilon^{s}\left(N_{S}\right)$ at small $N_{S}$ is too large for a DNS. In order to achieve reasonable accuracy it should be $N_{S}>2$, which confirms that the linear reverse interpolation, together with the other low-order methods, are not accurate enough to represent the back-reaction of the particles. The mild reduction of the spectrum for an increasing particle inertia could be a numerical artifact and will deserve further investigations.

It is interesting to characterize the statistics of the coupling term, in order to gain insight into the field that the forward NUFFT has to deal with. Particles that are close to each other may have very large velocity increments, compared to the fluid velocity increments. Hence, if one attempts to consider the particle velocity as a continuous field, averaging as in eqn (7), the resulting field would have large gradients. The field may also be multivalued, due to the ghost collision approximation. This non-smooth behaviour directly affects the coupling, since it depends on the two point statistics of the particle acceleration. The second order structure functions of the particle acceleration and the scaling exponents $\zeta_{a_{\|}}^{n}$ of the $n$-th order structure function of the particle acceleration are shown in Fig. 4. The second order structure functions display a well defined power law behaviour at small separation. However, the scaling exponent is much less than the value expected for a regular field. The exponents rapidly saturate at values which decrease with the particle inertia, confirming the irregularity of the coupling at small scales. Another issue for the representation of the coupling field is the particle clustering [24]. Inertial particles tend to sample strain-dominated regions of the flow agglomerating in those regions. As a consequence, the coupling term is a superposition of spikes that are not uniformly distributed in the domain.
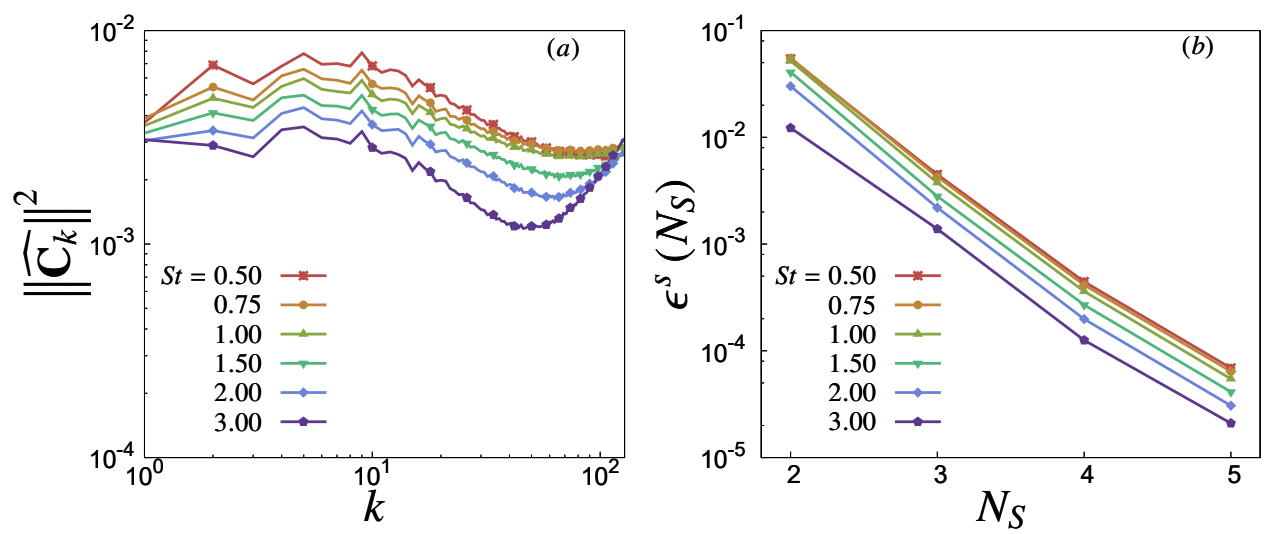

Figure 3: Spectral representation of the momentum coupling for different particle inertia. (a) Spectra of the momentum coupling computed with a 4th order basis; (b) Relative error in the computation of the spectrum of the momentum coupling by means of the NUFFT, with B-spline basis of different degree. 

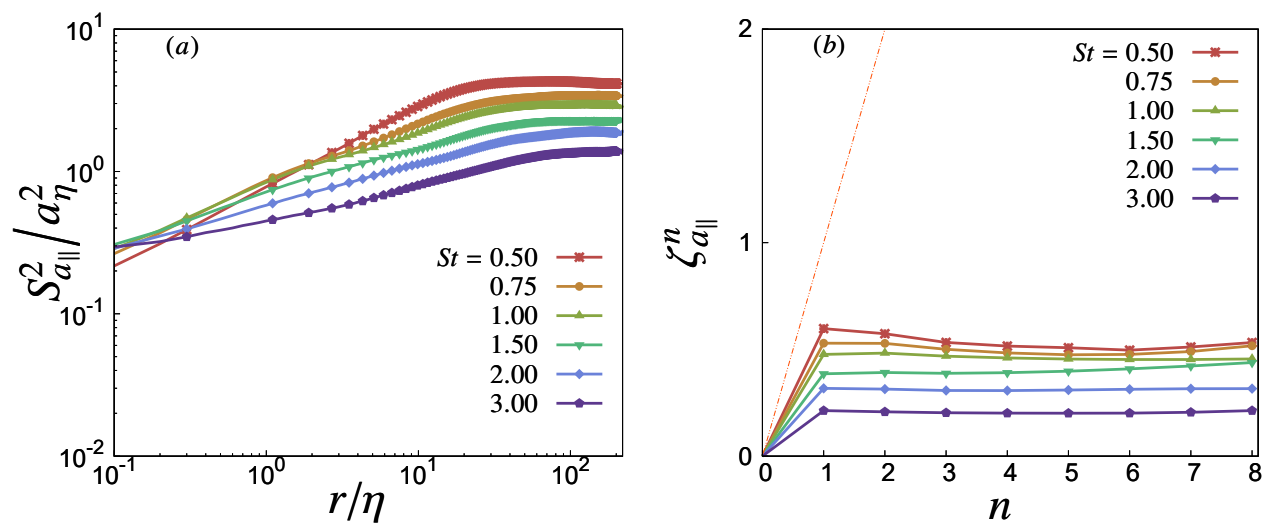

Figure 4: Structure functions of the particle acceleration for different particle inertia. (a) Second order structure functions of the particle acceleration; (b) Scaling exponent at small separation of the $n$-th order structure function of the particle acceleration. The statistics indicate the multifractal behaviour of the inertial particles.

\section{CONCLUDING REMARKS}

We have shown that the forward NUFFT provides an efficient and yet simple tool to obtain the accurate Fourier representation of the particle back-reaction on the flow. The forward NUFFT can be computed using the algorithm for the B-spline interpolation in reverse without introducing any additional computational load while keeping the limited support of the Bspline basis which allows an efficient parallelization of the method. We expect that the use of NUFFTs can lead to an improvement of the accuracy and efficiency of pseudo-spectral codes for two-way coupled particle-laden turbulent flows.

\section{ACKNOWLEDGEMENTS}

The authors thankfully acknowledge the computer resources provided by LaPalma Supercomputer at the Instituto de Astrof'1sica de Canarias through the Red Espa nola de Supercomputaci'on (project FI-2018-1-0044) and by CINECA under the ISCRA initiative, project HP10CWVISL. Additional resources were provided by HPC@POLITO (http://www.hpc.polito.it).

\section{REFERENCES}

[1] Zonta, F., Marchioli, C. \& Soldati, A., Direct numerical simulation of turbulent heat transfer modulation in micro-dispersed channel flow. Acta Mech, 195(1-4), pp. 305326, 2008.

[2] Ireland, P.J., Bragg, A.D. \& Collins, L.R., The effect of Reynolds number on inertial particle dynamics in isotropic turbulence. Part 1. Simulations without gravitational effects. J Fluid Mech, 796, pp. 617-658, 2016.

[3] Toschi, F. \& Bodenschatz, E., Lagrangian properties of particles in turbulence. Anпи Rev Fluid Mech, 41(1), pp. 375-404, 2009.

[4] Falkovich, G. \& Pumir, A., Sling effect in collisions of water droplets in turbulent clouds. J Atmos Sci, 64(12), pp. 4497-4505, 2007.

[5] Elghobashi, S., Particle-laden turbulent flows: direct simulation and closure models. Appl Sci Res, 48(3), pp. 301-314, 1991. 
[6] Yeung, P. \& Pope, S., An algorithm for tracking fluid particles in numerical simulations of homogeneous turbulence. J Comp Phys, 79(2), pp. 373-416, 1988.

[7] Hinsberg, van, M., Thije Boonkkamp, ten, J., Toschi, F. \& Clercx, H., On the efficiency and accuracy of interpolation methods for spectral codes. SIAM J Sci Comput, 34(4), pp. B479-B498, 2012.

[8] Gualtieri, P., Picano, F., Sardina, G. \& Casciola, C.M., Exact regularized point particle method for multiphase flows in the two-way coupling regime. J Fluid Mech, 773, pp. 520-561, 2015.

[9] Eggers, H., Knopp, T. \& Potts, D., Field inhomogeneity correction based on gridding reconstruction for magnetic resonance imaging. IEEE T Med Imaging, 26(3), pp. 374384, 2007.

[10] Toukmaji, A.Y. \& Board, J.A., Ewald summation techniques in perspective: a survey. Comp Phys Comm, 95(2), pp. 73-92, 1996.

[11] Pippig, M. \& Potts, D., Parallel three-dimensional nonequispaced fast fourier transforms and their application to particle simulation. SIAM J Sci Comput, 35(4), pp. C411-C437, 2013.

[12] Dutt, A. \& Rokhlin, V., Fast fourier transforms for nonequispaced data. SIAM J Sci Comput, 14(6), pp. 1368-1393, 1993.

[13] Greengard, L. \& Lee, J., Accelerating the nonuniform fast fourier transform. SIAM Review, 46(3), pp. 443-454, 2004.

[14] Sundaram, S. \& Collins, L.R., Numerical considerations in simulating a turbulent suspension of finite-volume particles. J Comp Phys, 124(2), pp. 337-350, 1996.

[15] Kumar, B., Schumacher, J. \& Shaw, R.A., Lagrangian mixing dynamics at the cloudyclear air interface. J Atmos Sci, 71(7), pp. 2564-2580, 2014.

[16] Zamansky, R., Coletti, F., Massot, M. \& Mani, A., Turbulent thermal convection driven by heated inertial particles. J Fluid Mech, 809, pp. 390-437, 2016.

[17] Pan, Y. \& Banerjee, S., Numerical simulation of particle interactions with wall turbulence. Phys Fluids, 8(10), pp. 2733-2755, 1996.

[18] Maxey, M.R. \& Riley, J.J., Equation of motion for a small rigid sphere in a nonuniform flow. Phys Fluids, 26(4), pp. 883-889, 1983.

[19] Canuto, C., Hussaini, M.Y., Quarteroni, A. \& Zang, T.A., Spectral Methods in Fluid Mechanics, Springer, 1988.

[20] Hochbruck, M. \& Ostermann, A., Exponential integrators. Acta Numer, 19, pp. 209286, 2010.

[21] Beylkin, G., On the fast fourier transform of functions with singularities. Appl Computat Harmonic A, 2(4), pp. 363-381, 1995.

[22] Pekurovsky, D., P3DFFT: a framework for parallel computations of Fourier transforms in three dimensions. SIAM J Sci Comput, 34(4), pp. C192-C209, 2012.

[23] van Hinsberg, M.A.T., Boonkkamp, J.H.M.t.T., Toschi, F. \& Clercx, H.J.H., Optimal interpolation schemes for particle tracking in turbulence. Phys Rev E, 87, p. 043307, 2013.

[24] Bragg, A.D. \& Collins, L.R., New insights from comparing statistical theories for inertial particles in turbulence: I. Spatial distribution of particles. New J Phys, 16(5), p. 055013, 2014. 


\title{
GLOBALLY VERSUS COMPACTLY SUPPORTED RBFS
}

\author{
EDWARD J. KANSA \\ Convergent Solutions, Livermore, USA
}

\begin{abstract}
For many years, a debate has occurred whether radial basis functions having compact support (CS) or global support (GS) is best for engineering and scientific applications. CS RBFs converge as $\mathrm{O}\left(\mathrm{h}^{(\mathrm{k}+1)}\right)$, $\mathrm{h}$ is the fill distance, and its systems of equations have many zeros. In contrast, GS RBFs converge as $\mathrm{O}(\lambda(\mathrm{c} / \mathrm{h})), \lambda<1, \mathrm{c}$ is the GS-RBF shape parameter. Previously, the barrier to exploiting the exponential convergence rate of GS-RBFs has been the ill-conditioning problem that is due to computer chip restrictions on the relatively large machine epsilon. Although computer chips with arbitrary precision are very rare presently, extended precision software has allowed the exploitation of the exponential convergence rates of GS-RBFs. When attempting modeling of higher dimension practical problems, previous methods such as domain decomposition, global optimization, pre-conditioning will need to be blended even on massively parallel computers.

Keywords: radial basis functions, compact support, global support, polynomial convergence, exponential convergence, multi-precision arithmetic.
\end{abstract}

\section{INTRODUCTION}

Kansa [1], [2] presented a meshless radial basis function (RBF) to solve partial differential equations (PDEs). There are two approaches to solve partial differential and integral equations with meshless RBF methods: 1. "Compactly supported" RBFs (CS-RBFs); 2. "Globally supported" (GS-RBFs). The convergence rate for interpolation is $\lambda^{(\mathrm{c} / \mathrm{h})}$, where $\lambda<1$ [3]-[5]. The ratio, c/h, increases the rate of convergence by decreasing $h$, the average fill distance between pairs of points (h-refinement) or increasing the average value of the shape parameter, c, (c-refinement). The basic question is for a given application is whether hrefinement or c-refinement is more cost effective to achieve the target accuracy with the least computational effort. The answer to this question is not definitive but depends upon the nature of the application and the available resources.

In general, as system of equations becomes larger, the higher is the probability that rounding-errors will severely contaminate the solution. A measure of this severity is the condition number, $\kappa(\mathbf{A})$, of a system of equations relative to the inverse machine epsilon, $1 / \varepsilon_{\mathrm{M}}$. Whenever $\kappa(\mathbf{A})>1 / \varepsilon_{\mathrm{M}}$ the solution is worthless. While it is impossible to construct an ideal Platonic computer with infinite precision, infinite memory capacity that operates infinitely fast, there are some procedures that can be adopted to control the damage caused by the accumulation of round-off errors on a finite precision computer. Increasing the number of digits of precision requires both sacrificing memory and increasing the processing time per unit operation. This sacrifice is worthwhile if the total processing time to achieve target accuracy is smaller than the total processing time with single or double precision.

\section{TREATING ILL-CONDITING}

More or less standard methods have been used to treat either CS-RBF or GS-RBF systems such are regularization in which a small positive value is added to the diagonal elements (Tikhonov [6], Tikhonov and Arsenin [7], Tikhonov et al. [8]). Also related, is the truncated singular value decomposition (SVD) m0ethod in which the singular values below a certain threshold are neglected, and a modified set of linear equations is used to find the expansion 
coefficients. As successively more small singular values are deleted. The further does the modified matrix deviate from the original matrix or set of linear or linear set of equations.

Holobodrodko of the Advanpix LLC [9] consider the eigenvalues of a 150x150 symmetric Grcar matrix [10] whose condition number is 3.61. Obviously, a very low condition symmetric matrix ought to have very accurate eigenvalues; and it is obvious that the eigenvalues of both the Grcar matrix and its transpose ought to be identical. However, the Grcar matrix whose condition number is 3.61 , on a double precision machine, produces incorrect, unequal eigenvalues. Only when the extended precision software package is used do the eigenvalues in a quadruple precision calculation become identical. As a cautionary note, ideal Platonic arithmetic and mathematics does exist on real computers; rather, fuzzy arithmetic and mathematics is perhaps more appropriate, with the fuzziness dependent upon the available precision on the computer.

The reproducing kernel based Gaussian method expands a Gaussian RBF as an eigenfunction of products of the Gaussian exponentials in $\mathfrak{R}^{\mathrm{n}}$, truncated to a finite number of terms, M [11], [12]. The kernel expansion is still ill-conditioned, so a combination of a truncated SVD and the quotient residual (QR) methods are used to obtain a new set of basis functions. The change of that is much better conditioned for h-refinement.

Domain decomposition has been successfully to both CS-RBF and GS-RBF methods for PDEs. Domain decomposition can use either the overlapping or non-overlapping methods [13]-[19]. Assume the problem is continuous, and the computational domain is arbitrarily decomposed as a union of smaller subdomains containing an equal number of data centers (approximately). Just as with finite element methods, the larger the number of centers within a subdomain, the larger will the condition number be, either with CS-RBFs or GS-RBFs. The IE or PDE in each subdomain has its "boundary" and interior conditions. In each subdomain, the set of equations is solved on separate independent processors in parallel. Then the solutions in each subdomain are iteratively blended to enforce continuity of the function and its normal and tangential derivatives. Typically, only 2-3 iterations are required to obtain satisfactory blending convergence. However, the parallelism is somewhat different if compact or global support is used; this will be discussed later.

If the problem being solved is fairly homogeneous spatially, then increasing the shape parameters or c-refinement while increasing the arithmetic precision is vastly more efficient since the total number of data centers can be reduced by many orders of magnitude [20]. However, some problems may have so much fine scale structure that h-refinement, rather than c-refinement, is required and slower converging compactly supported RBF methods may be required for a given available computer. Common sense is required regarding how to approach a given problem using the available resources.

Very promising is the development of global optimization methods for solving systems of equations [21]-[28]. This is a very broad field of research and development that has the potential to solve large systems of both linear and nonlinear equations without the need for LU methods that exacerbates ill-conditioning. Global optimization can be either search procedures, stochastic procedures, or a hybrid mixture of both.

\section{RECOMMENDED STRATEGIES}

It is recommended here that a hybrid approach be used such as: matrix scaling, iterative refinement, pre-conditioning, domain decomposition, global optimization, and extended arithmetic precision. Higham [29] discussed the importance of multiple precision in the practical world, and specifically praised the work of Holobodroko for the development of the multi-precision software package [9]. 
In addition, the curse of dimensionality is a serious impediment to h-refinement because of memory storage issues even on the most recent massively parallel computers. Plasma physics is minimally a six-dimensional problem. Discrediting at 1,000 points/dimension requires a storage capacity of $10^{18}$ for just the data centers alone.

A hardware computer chip remedy for extended precision beyond 64 bits/word is most preferable. However, the consumer gadget market does not require much precision, unlike scientific computing. For scientific applications, only software methods are the only current method to obtain very high precision. The Advanpix mufti-precision computing toolbox (AMCT) concretely demonstrates that multi-precision arithmetic, if properly constructed, can be executed very efficiently, unlike other software packages for multi-precision calculations. The exponential convergence rate becomes vital in solving multi-dimensional (many independent variables).

It is important to present the case for GS-RBF methods for large scale, multi-bimensal IE and PDE problems that will be executed on parallel computers. Some tools for massively parallel computers are being developed in the languages, $\mathrm{C}$ and $\mathrm{C}++$ [30], "The GNU Multiple Precision Arithmetic Library". At first glance, CS-RBFs are very appealing because the matrix associated with calculating the expansion coefficients is sparse.

The performance of direct solvers for sparse matrices using LU, CHOL, and other solvers, depends entirely on the locations of the non-zero elements. Bad locations of the zeros can require a full $\mathrm{O}\left(\mathrm{N}^{3}\right)$ computational complexity. To alleviate this complexity problem, all algorithms use matrix pre-ordering to minimize the fill-in; the time required for pre-ordering must be considered. The optimal pre-ordering algorithm is known only for symmetricpositive definite matrices. But, in the case of unsymmetrical matrices, optimal pre-ordering is an NP-complete task.

All the existing direct solvers for sparse systems use some kind of heuristic ideas to perform the pre-ordering. The complexity of direct solvers for sparse matrices can vary wildly from $\mathrm{O}\left(\mathrm{N}^{3 / 2}\right)$ to $\mathrm{O}\left(\mathrm{N}^{3}\right)$. Direct sparse solvers have poor capability for parallel execution, whereas dense solvers are extremely suitable for parallelism. A discussion of the sparse complexity issues can be found at www.mathworks.com/help/matlab/math/sparsematrix-operations.html.

Theoretically, iterative methods are the only way to beat the complexity of direct solvers since iterative solvers are supposed to converge within $\mathrm{O}\left(\mathrm{N}^{2}\right)$ operations. However, iterative solvers suffer from slow convergence and good pre-conditioners are required, but finding good pre-conditioners is separate problem that needs to be solved. It is possible that with careful and non-trivial tuning of all parts of sparse solvers (direct or iterative) can be faster than solving small dense matrix in ex studied and the computational environment for comparison.

Both GPUs (graphic processing units) and FPG tended precision on a single CPU. However, everything depends on the particular problem being A (field-programmable gate arrays) possess highly parallel structures that makes them more efficient than generalpurpose CPUs (central processing units) for algorithms where the processing of large blocks of data is performed in parallel. However, GPUs and FPGAs have very limited functionality for branching operations that are abundant in sparse solvers making it very difficult for porting and running in parallel.

On the contrary, dense solvers enjoy massive parallelism because there is no branching and all manipulations can be formulated as efficient BLAS3/GEMM operations that are well optimized and are designed for massive parallelism on all imaginable platforms.

If the Advanpix toolbox is executed for full solvers on CPUs with 10 or more cores, or GPUs - the toolbox will beat sparse-based algorithms. The justification for this claim is that 
dense solvers in the Advanpix toolbox are well suited for parallel execution whereas sparse solvers are not. Intuitive conclusions about the efficiency of sparse versus dense RBF systems can be misleading. In addition, sparse methods only enjoy polynomial convergence rates whereas global RBF systems enjoy exponential convergence rates.

\section{REFERENCES}

[1] Kansa, E.J., Multiquadrics: A scattered data approximation scheme with applications to computational fluid dynamics I: Surface approximations and partial derivative estimates. Comput. Math. Applic., 19(8/9), pp. 127-145, 1990.

[2] Kansa, E.J., Multiquadrics: A scattered data approximation scheme with applications to computational fluid dynamics II: Solutions to parabolic, hyperbolic, and elliptic partial differential equations. Comput. Math. Applic., 19(8/9), pp. 147-161, 1990.

[3] Madych, W.R. \& Nelson, S.A., Multivariate interpolation and conditionally positive definite functions ii. Math. Comp., 54, pp. 211-230, 1990.

[4] Madych, W.R., Miscellaneous error bounds for multiquadric and related interpolators. Com-put. Math. Appl., 24(12), pp. 121-138, 1992.

[5] Madych, W.R. \& Nelson, S.A., Bounds on multivariate polynomials and exponential error estimates for multiquadric interpolation. J. Approx. Theo., 60, pp. 94-114, 1992.

[6] Tikhonov, A.N., On the stability of inverse problems. Dokl.Akad.i Nauk SSSR, 39(5), pp. 195-198, 1943.

[7] Tikhonov, A.N. \& Arsenin, V.Y., Solution of Ill-posed Problems, Winston \& Sons: Washington, 1977.

[8] Tikhonov, N., Goncharsky, A.V., Stepanov, V.V. \& Yagola, A.G., Numerical Methods for the Solution of Ill-Posed Problems, Kluwer Academic Publishers, 1995.

[9] Advanpix LLC., Multiprecision computing toolbox for MATLAB, Yokohama, Japan, 2008-2017. www.advanpix.com.

[10] Grcar, J.F., Operator coefficient methods for linear equations. Technical Report SAND89-8691, Sandia National Labs, 1989.

[11] Fasshauer, G.E., Hickernell, F.J. \& Wozniakowski, H., Rate of convergence and tractability of the radial function approximation problem. SIAM J. Numer. Anal., 50(1), pp. 247-271, 2012.

[12] Fasshauer, G.E. \& McCourt, M.J., Stable evaluation of Gaussian RBF interpellants. SIAM J. Scient. Comput., 34(2), pp. A737-A762, 2012.

[13] Hon, Y.-C. \& Wu, Z., Additive Schwarz domain decomposition with radial basis approximation. Int. J. Appl. Math. Stat., 4, pp. 81-98, 2002.

[14] Li, J. \& Hon, Y.-C., Domain decomposition for radial basis meshless methods. Num. Meth. PDEs, 20, pp. 450-462, 2004.

[15] Ingber, M.A., Chen, C.-S. \& Tanski, A., A mesh free approach using radial basis functions and parallel domain decomposition for solving three-dimensional diffusion equations. Int. J. Num. Meth. Eng., 60, pp. 2183-2201, 2004.

[16] Duan, Y., Tang, P.F., Huang, T.Z. \& Lai, S.J., Coupling projection domain decomposition method and Kansa's method in electrostatic problems. Comput. Phys. Commun., 180, pp. 200-214, 2009.

[17] Beatson, R.K., Light, W.A. \& Billings, S., Fast solution of the radial basis function interpolation equations: Domain decomposition methods. SIAM J. Sci. Comput., 22, pp. 1717-1740, 2006.

[18] Hernandez-Rosales, A. \& Power, H., Non-overlapping domain decomposition algorithm for the Hermite radial basis function meshless collocation approach: 
applications to convection diffusion problems. J. Algo. \& Comput. Tech., 1, pp. 127159, 2007.

[19] Cavoretto, R., De Rossi, A., Donatelli, M. \& Serra-Capizzano, S., Spectral analysis and preconditioning techniques for radial basis function collocation matrices. Numer. Lin. Alg. Appl., 19, pp. 31-52, 2012.

[20] Galperin, E.A. \& Kansa, E.J., Application of global optimization and radial basis functions to numerical solutions of weakly singular Volterra integral equations. Comput. Math. Appl., 43(3-5), pp. 491-499, 2002.

[21] Skalna, I., A global optimization method for solving parametric linear systems whose input data are rational functions of interval parameters, Lecture Notes in Computer Science, 6068, Springer: Berlin, Heidelberg. ISBN: 978-3-642-14402-8.

[22] Mafteiu-Scai, L.O. \& Mafteiu-Scai, E.J., Solving linear systems of equations using a memetic algorithm. Int. J. Comput. Applic., 58, pp. 16-22, 2012.

[23] Taheri, S. \& Mammadov, M., Solving systems of nonlinear equations using a globally convergent optimization algorithm. Global J. Tech. \& Optim., 3, pp. 132-138, 2012.

[24] Kaeck, J.E., Constrained global optimization with radial basis functions. Research Report MdH-IMa-2004, Department of Mathematics and Physics, Malardalen University, Sweden.

[25] Bjorkman, M. \& Holmstroem, K., Global optimization of costly nonconvex functions using radial basis functions. Optim. \& Eng., 1, pp. 373-397, 2000.

[26] Gutmann, H.M., A radial basis function method for global optimization. J. Global Optim., 19, pp. 201-227, 2001.

[27] Le Thi, H.A., Vaz, A.I.F. \& Vicente, L.N., Optimizing radial basis functions by DC programming and its use in direct search for global derivative-free optimization. TOP, 20, pp. 190-214, 2012.

[28] Jakobsson, P., Patriksson, M., Rudholm, J. \& Wojciechowski, A., A method for simulation based optimization using radial basis functions. Opt. \& Eng., 11, pp. 501$532,2010$.

[29] Higham, N.J., Multi-precision world. SIAM News, pp. 2-3, Oct. 2017.

[30] The GNU Multiple Precision Arithmetic Library. https://gmplib.org/. 